\title{
MACRE: A Novel Distributed Congestion Control Algorithm in DTN
}

\author{
Ying $A n^{1, a}$, Xi Luo $2, b$ \\ ${ }^{1}$ College of Computer Science and Technology, Hunan International Economics University, \\ Changsha, China; \\ School of Information Science and Engineering, Central South University, Changsha, China. \\ ${ }^{2}$ Department of Computer Science and Technology, Hunan Police Academy, Changsha, China; \\ School of Information Science and Engineering, Central South University, Changsha, China. \\ aanan1428@163.com, bhnluoxi@163.com
}

Keywords: DTN, congestion control, admission control

\begin{abstract}
Congestion control is a very important problem which can affect network performance directly. However, owing to the lack of steady end-to-end connection and high latency, the traditional congestion control mechanism based on end-to-end feedback is not feasible in DTN. Because obtaining the global information of network is difficult, the congestion control decisions should be made autonomously with local information only. We propose a novel distributed congestion control algorithm based on epidemic routing protocols----MACRE (Message Admission Control based on Rate Estimation). Preliminary experimental results show that this congestion control mechanism can improve the network performance efficiently.
\end{abstract}

\section{Instruction}

DTN is a new kind of network which has bright characteristics: high delay and low data rate, no steady existence of link between end to end, lacking of interaction in the network architecture, the system resources and the life time is limited and so on. Therefore, this kind network also be called as challenged network $^{[1]}$. In order to overcome the restrictions mentioned above, sustain all kinds of applications in DTN better, DTN adopts "storage-carry-forward" routing ${ }^{[2]}$ to take place of traditional "storage-forward" routing. Because of the instability of the end-to-end path, the replication of messages is a normal method to ensure the delivery ratio of messages. Typical routing protocol such as epidemic routing protocol ${ }^{[3]}$ can improve the delivery ratio by increasing the amount of repetitions of messages and the forward opportunity of messages. However this kind of mechanism will bring large number of repetitions, and even when the messages have been successfully received by the destination, a lot of redundant repetitions will be stored in the network yet. Then the limited resources of network will be exhausted rapidly by the gigantic amount of repetitions, which will result in network congestion and decline of the delivery ratio finally. Consequently, how to avoid and control network congestion efficiently has became a key problem to improve the performance of network。

\section{Related Works}

Congestion control is a new research field in DTN, compared with that of traditional network, congestion control in DTN is more difficult. The lack of steady end-to-end path and the dynamic characteristics of DTN make the traditional congestion control policy based on feedback in TCP/IP is not applicable in delay/interrupt tolerant environment.

Congestion control mechanism can be divided into Proactive Policy and Reactive Policy ${ }^{[4]}$. Proactive Policy usually applies admission control to avoid network congestion at the beggining. Scott Burleigh presents a local autonomy congestion control mechanism based on rules ${ }^{[5]}$. This mechanism adopts economics model and compares the receipt and forwarding of messages to risk investment. When a new message arrives, node independently decides whehter to receive it or not according to a risk value of receiving and storing the message instead of the end-to-end feedback 
information. The risk value is determined by several factors, such as the node's own buffer space, the input data rate, the residual TTL of message and so on. The advantage of this mechaninsm is each router can make independent decision based on local information and prevent congestion to a certain extent.

On the contrary, Reactive Policy performs some response actions when the congestion has occurred. The typical response mode is executing message drop policy. Reference ${ }^{[6]}$ proposes DRA algorithm which drops the messages stored in the buffer randomly when congestion occurred. This algorithm is so simple that its effection is limited.

Reference $^{[7]}$ proposed DO (Drop-Oldest), DY (Drop-Youngest), DF (Drop-Front) and DL (Drop-Last) algorithm to remit congestion by releasing buffer spaces. DO algorithm drops the oldest message whose residual TTL is shortest when congestion occurred at the node. The basic idea of DO is the oldest message in the network has been received by destination most probably. On the contrary , DY algorthm drops the youngest message when congestion occurred at the node, because DY believes the message whose residual TTL is longest has the greater probability to be forwarded by the other nodes to the destination in the future. DF drops the front message that stayed in the buffer for the longest time when congestion occurred at the node. According to the logical reasoning, there is in all probabilty that the message has been forwarded to other nodes, in other words, this message has great probability to be carried and replicated by other nodes and forwarded to the destination finally. On the contrary of DF, DL drops the last message that stayed in the buffer for the shortest time when congestion occurred at the node. DL made the messages who came into the buffer firstly have more opportunities to be forwarded out, especially when using FIFO forward mechanism. Simuliar mechanisms include ${ }^{[8-11]}$, but these mechanisms may make the performance of network declined.

Furthermore, Juan Alonso and Kevin Fall proposed a linear programming method which called $\mathrm{LP}^{[12]}$ to solve network congestion under the circumstance that the route is determined. But this mechanism was executed on the hypothesis that the link capacity, transmission delay and the change of network topology is predictable. In addition, the cost of this mechanism is very high.

Based on analyzing the existing congestion control mechanisms, taking the characteristic of DTN into account, this paper proposes an adaptive distributed congestion control algorithm-Message Admission Control based on Rate Estimation (MACRE). When a new message arrives, the nodes execute admission control according to the occupancy ratio of its own buffer space and the historical statistics mean value of input/output rate. This algorithm can efficiently prevent the buffer of nodes be consumed excessively and then avoid the congestion occurring.

\section{Message Admission Control based on Rate Estimation (MACRE)}

The simplest admission control policy is determining whether receive the new message according to the free buffer size of node. While in some cases, it is not suitable to make a decision simply based on the buffer size of node. For example, the life time of message is limited, it is restricted by TTL. When a message arrives, if its residual TTL is not enough to send out the message, the message also should be refused even if the node buffer is enough to contain it. Furthermore, when the node buffer space is abundant, the node should receive as many messages as its possible, consequently providing transmission service for more messages. Moreover, a strict admission control, which is based on the current network status, becomes imperative when the node buffer space is severely limited. In detail, when the occupancy ratio of node buffer is close to the threshold value (node is in the critical state of network congestion), if the messages stored in buffer can not be transmitted to release sufficient buffer space, the resources will be exhausted by the new arrival messages, and sequentially lead to network congestion. So we propose a novel congestion control algorithm—-Message Admission Control based on Rate Estimation (MACRE), which can make the decision whether admit a message according to the relationship between input rate and output rate of node. Namely, when a new message arrives, if the input rate less than the output rate, it implies the occupancy ratio of node buffer will appear net decrease (the residual free buffer space will be further 
increased), then node receives the message, otherwise refuses it. Obviously, the instantaneous input/output rate may not reflect the fact of network status, thus, we choose the historical statistical mean value of data rate instead of the instantaneous data rate. The calculation method is shown in Eq.(1):

$$
R_{i}=\alpha \cdot \mathrm{R}_{\text {sample }}+(1-\alpha) R_{i-1}
$$

Here, Rsample denotes the sampling value of current data rate; Ri-1 denotes the former historical statistical mean value of data rate; Ri denotes the current value; $\alpha \in(0,1)$ is the weighted smoothing factor.

Our algorithm is described as follows:

$\boldsymbol{N}$ — the size of the arrival message;

$\boldsymbol{K}$ — the buffer capacity of node;

freeBuffer — the free buffer size of node;

$\boldsymbol{R}_{\boldsymbol{i n}}$ - the historical statistical mean value of data input rate;

$\boldsymbol{R}_{\text {out }}$ the historical statistical mean value of data output rate;

$\boldsymbol{R T T L} \longrightarrow$ residual time to live of message;

$\boldsymbol{\beta}-a$ constant, $\beta \in(0,1)$.

Algorithm. Node ProcessEvent(message $M$ arrives)

1: if $N>$ freeBuffer $\| R_{\text {out }} \times R T T L<N$ then

2: $\operatorname{discard} M$;

3: else if freeBuffer $>\beta \times K$ then

4: receive $M$;

5: else if $\left(R_{\text {in }}-R_{\text {out }}\right) \times R T T L<$ freeBuffer then

6: receive $M$;

7: else

8: $\operatorname{discard} M$;

\section{Evaluation}

\section{A. Experimental Set-up}

To evaluate our algorithm, the ONE (Opportunistic Networking Environment) simulator ${ }^{[13]}$ is used for our simulators on the mobile model of the Random Waypoint model. The size of network is $4500 \mathrm{~m} \times 3400 \mathrm{~m}$, nodes are randomly placed in the network and these nodes move randomly at speeds of $0-25 \mathrm{~m} / \mathrm{s}$. The communication distance of node is 150 meters, and data transmission speed is $2 \mathrm{Mbps}$. The sender and the receiver are all randomly selected from those network nodes. Each sender sends one message every 25 35 seconds. The TTL of messages is 6000s. The simulation time is 24 hours. And the Epidemic Routing algorithm is adopted in our experiment.

\section{B. Performance Evaluation}

In this section, we will evaluate the performance in terms of delivery ratio, and throughput. In our paper, the delivery ratio is defined as the ratio of number of packets received by destination nodes to the number of packets sent by source nodes, which is shown in Eq. (2). And the throughput is defined as all the messages successfully received by destination nodes per second, which is shown in Eq. (3). We ran the Epidemic routing protocol with and without congestion control to demonstrate the benefits of our congestion control algorithm. To simulate varying degrees congestion we decrease the size of the node buffer, and compare the performance metrics mentioned above. 


$$
\begin{aligned}
& \text { delivery ratio }=\frac{\text { number of packets received }}{\text { number of packets sent }} \times 100 \% \\
& \text { throughput }=\frac{\text { number of messages successfully received by the destination }}{\text { sampling duration }}
\end{aligned}
$$

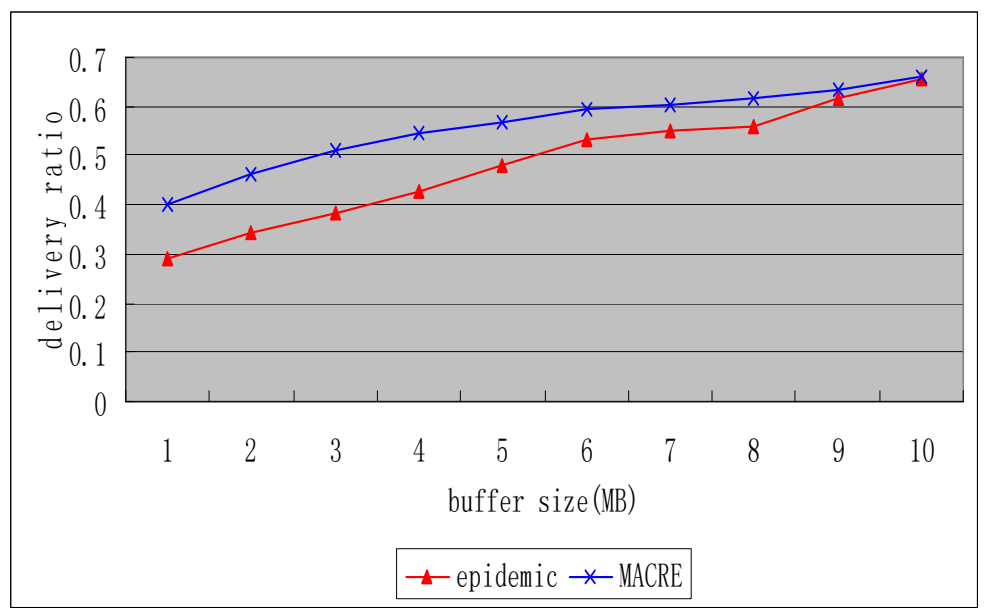

Fig.1 Delivery ratio comparison between Epidemic Routing and MACRE for increasing buffer size

Obviously, the delivery ratio increases with the increasing of buffer size. And when the network congestion status is serious (node buffer size is small), the delivery ratio of MACRE is remarkably higher than original epidemic routing as shown in Fig.1. Of course, we can also find out that the delivery ratio of epidemic routing increases near to that of MACRE with the increasing of buffer size.

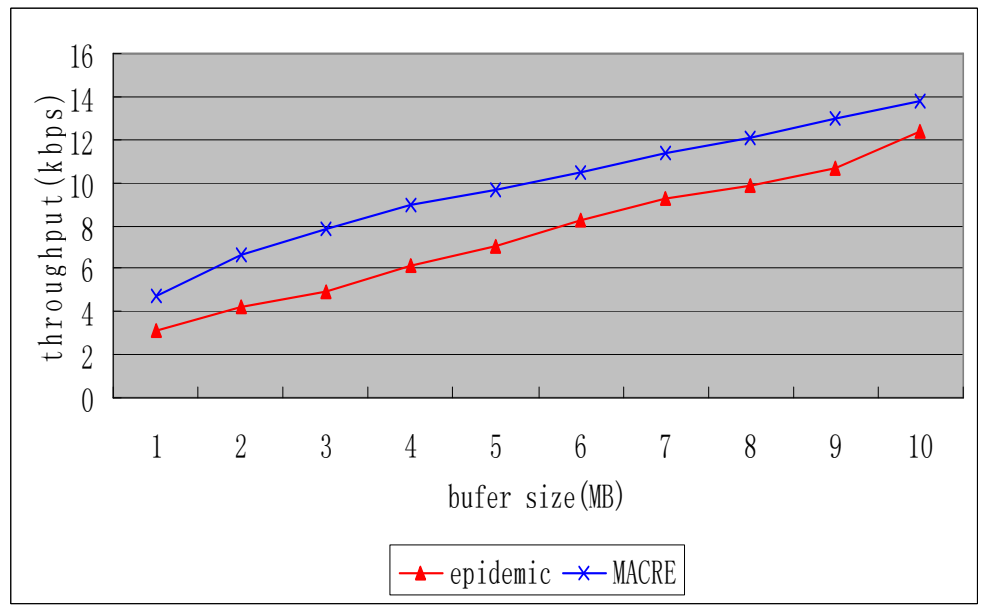

Fig.2. Throughput comparison between Epidemic Routing and MACRE for increasing buffer size

In Fig.2, it is easy to see that the throughput of MACRE is about 20 percent higher than that of epidemic routing even if the network is badly congested. The results of our simulation show that MACRE is effective to congestion control in DTN.

\section{Conclusion}

Delay Tolerant Networks (DTN) is a special network, in which end-to-end connectivity may not exist continuously and latency will be high. In order to achieve data delivery, store-carry-forward protocols are used in DTN and routing protocols based on epidemic message dissemination has been proposed, such as Epidemic routing. But the resources of DTN is very limited, message replication may rapidly overwhelm node resources, so network congestion may occur easily. However, owing to the characteristics of DTN, the traditional congestion control mechanism based on end-to-end feedback is not feasible. We present a novel distributed congestion control algorithm based on epidemic routing protocols----MACRE (Message Admission Control based on Rate Estimation), which autonomously makes the decision whether admit a message according to the relationship between input rate and output rate of node. We show through simulation that our algorithm effectively improves delivery rates in some scenarios and obtains high throughput. 


\section{Reference:}

[1] Kevin Fall , "A Delay-Tolerant Network Architecture for Challenged Internets", in Proceedings of SIGCOMM'03,Aug.2003.

[2] Luciana Pelusi, Andrea Passarella, Marco Conti. Opportunistic Networking: Data Forwarding in Disconnected Mobile Ad hoc Networks. IEEE Communications Magazine .November 2006.

[3] A. Vahdat and D. Becker. Epidemic routing for partially connected ad hoc networks. Technical Report CS-200006, Duke University, April 2000.

[4] Fan Xiumei,Shan Zhiguang,Zhang Baoxian et al. State-of-the-art of the architecture and techniques for delay tolerant networks[J].Chinese Journal of Electronics,2008,36(1):161-170(in Chinese).

[5] Burleigh, E. Jennings, J. Schoolcraft. Autonomous Congestion Control in Delay-Tolerant Networks. In proc. of 9th International Conference on Space Operations. 2006.

[6] James A. Davis, Andrew H. Fagg, Brian N. Levine. Wearable Computers as Packet Transport Mechanisms in Highly-Partitioned Ad Hoc Networks. IEEE. 2001. 141-148

[7] A.Lindgren and K.S.Phanse. Evaluation of queuing policies and forwarding strategies for routing in interrmittently connected networks. In Proc. of IEEE COMSWARE. January 2006.

[8] M. Seligman, K. Fall and P. Mundur. Alternative Custodians for Congestion Control in Delay Tolerant Networks", in Proc. of ACM SIGCOMM, 2006.

[9] Storage Usage of Custody Transfer in Delay Tolerant Networks with Intermittent Connectivity , Matthew Seligman, 2006.

[10] Selgman. M, Fall. K, Mundur. P. Storage Routing for DTN Congestion Control. Wireless Communications and Mobile Computing, v 7, n 10, p 1183-96, Dec. 2007.

[11] Daowen Hua, Xuehui Du, Guoyu Xu, Lifeng Cao, Huan Chen, A DTN Congestion Mechanism Based on Distributed Storage. 2010 2nd IEEE International Conference on Information Management and Engineering (ICIME 2010), p385-9, 2010.

[12] Juan Alonso, Kevin Fall, A Linear Programming Formulation of Flows over Time with Piecewise Constant Capacity and Transit Times. IRB-TR-03-007.

[13] A. Ker"anen, J. Ott, and T. K"arkk"ainen, The one simulator for dtn protocol evaluation, in Simutools, 2009. 